\title{
Clock Gene Timeless in the Chagas Disease Vector Triatoma infestans (Hemiptera: Reduviidae)
}

\author{
María M. Stroppa* and Beatriz A. García \\ Instituto de Investigaciones en Ciencias de la Salud (INICSA), CONICET and Cátedra de Bioquímica y Biología Molecular, Facultad de Ciencias \\ Médicas, Universidad Nacional de Córdoba, Córdoba, Argentina
}

\begin{abstract}
To contribute to a better understanding of the molecular basis of the circadian biological rhythms in Chagas disease vectors, in this work we identified functional domains in the sequences of the clock protein TIMELESS (TIM) in Rhodnius prolixus and analyzed the expression of the timeless (tim) gene at the mRNA level in Triatoma infestans. The tim gene expression in nervous tissue of adult $T$. infestans revealed clear oscillations in the abundance of the transcript in both sexes in the group maintained under photoperiod with a daily canonical rhythm, showing a significant increase in expression at sunset. As expected, in the group maintained in constant light, no daily increase was detected in the tim transcript level.
\end{abstract}

\section{INTRODUCTION}

Triatoma infestans (Hemiptera, Reduviidae) is the main vector of Chagas disease in the Southern Cone of Latin America. Vector control has proven to be difficult, and, recently, insecticide resistance has emerged as one of the main explanations of unsatisfactory control observed. ${ }^{1}$ Chronobiological studies could be a novel and relevant aspect that might contribute to the development of more effective control programs. Endogenous or circadian clocks make the temporary coordination between biological processes and environmental cycles. Among the most important external signals or synchronizers (Zeitgebers) are light/dark alternation, the variation in environmental temperature, the availability of food, and social interaction. ${ }^{2-4}$ In Drosophila melanogaster, the clock is composed of two "loops" interrelated by feedback, period/timeless loop (per/tim loop), and clock loop, and encoded by the period (per), timeless (tim), clock (Clk), and cycle (cyc) genes. ${ }^{5}$ Homologous circadian genes are found in all insect clocks, but their contribution to species-specific circadian timing systems differs.

Circadian rhythms in biological processes such as reproduction, foraging, breeding, oviposition, dispersion, and host seeking have been extensively studied in adults of Rhodnius prolixus and T. infestans. ${ }^{6-11}$ In a previous work, the per gene expression showed a daily canonical rhythm in nervous tissue of groups of adult individuals of $T$. infestans maintained under photoperiod or light/dark cycle (LD) and constant dark. ${ }^{12}$ With the propose to analyze the circadian expression profile of the tim gene in $T$. infestans, in this work the tim mRNA level in nervous tissue of adults specimens was determined under different dark/light regimes.

\section{MATERIALS AND METHODS}

Triatoma infestans were reared at $28 \pm 1^{\circ} \mathrm{C}$ at a relative humidity of 60-70\% and fed once every 2 weeks on restrained chickens. Since the fifth-instar nymph stage, $T$. infestans individuals were maintained under different dark/light regimes.

\footnotetext{
* Address correspondence to María M. Stroppa, Instituto de Investigaciones en Ciencias de la Salud, Cátedra de Bioquímica y Biología Molecular (CONICET-UNC), Facultad de Ciencias Médicas, Universidad Nacional de Córdoba, Pabellón Argentina, 2do Piso, Ciudad Universitaria, Córdoba 5000, Argentina. E-mail: mercedesstroppa@ hotmail.com
}

Two experimental groups were subjected either to 1) light/dark cycle (LD) or 2) constant light (LL). The LD cycle group consists of 12 hours of light and 12 hours of darkness. Time of day was reported in 24-hour Zeitgeber time (ZT), with ZT12 (20:00 hours) defined as time of lights off and ZT0 (08:00 hours) defined as end of the dawn transition under the LD cycle. For the LL group, subjective day was reported between ZTO (8:00 hours) and ZT12 (20:00 hours). For the circadian study of the tim gene expression, the heads (brain nervous tissue) were excised between 40 and 45 days after molt from adult female and male specimens of the two experimental groups every 4 hours over 24 hours. In all cases, the tissues were dissected under aseptic conditions and stored in liquid nitrogen until used for RNA extraction.

Total RNA was isolated from pools of insect tissues using MasterPure RNA Purification Kit (Epicentre, Madison, WI). Samples from five adult females or five adult males were pooled. Synthesis of cDNA was performed from the total RNA using Oligo- $\mathrm{dT}_{20}$ and SuperScript III Reverse Transcriptase (Invitrogen, Carlsbad, CA). The amplification of a fragment of the tim gene (200 base pairs) was carried out by the polymerase chain reaction (PCR) using the primers forward ATTTGGTTGGAACAAAGCTGAT and reverse TGAAGGTGT TGCTTTATGTGAAC. After the PCR products were purified, they were sent for direct sequencing to the Genomics Unit/ Node CATG National Genomics Platform, Institute of Biotechnology (CICVyA-INTA's, Buenos Aires, Argentina). The transcript levels of the tim gen were measured by semiquantitative PCR and expressed as described in a previous work carried out in our laboratory. ${ }^{12}$ The experimental values represent mean $\pm S D$ of two independent experiments for each sample composed by pooled tissues from five specimens. The significance of differences in tim transcript gene levels was determined using analysis of variance. All statistical calculations were performed using Prism 5 software (GraphPad, San Diego, CA). The rhythms in tim mRNA expression were analyzed using the JTK_Cycle nonparametric algorithm. ${ }^{13}$ In this statistical analysis, the $P$-value, period, phase (LAG), and amplitude (AMP) were evaluated.

To identify conserved functional domains, an alignment was carried out with the deduced TIMELESS (TIM) protein sequences from $R$. prolixus Contig 3.0.3-177.8 (GeneBank: ACPB03012942.1) and those from Papilio xuthus (GenBank: KPI93841.1), Ephestia kuehniella (GenBank: AJG06482.1), Antheraea pemyi (GenBank: AAF66996.1), Tribolium castaneum 
(GenBank: AOR83338.1), D. melanogaster (GenBank: NP 001334730.1), Aedes aegypti (GenBank: AAY40757.1), Belgica antarctica (GenBank: AGZ88039.1), Clunio marinus (GenBank: AFS34623.1), Halyomorpha halys (GenBank: XP_014278196.1), and Cimex lectularius (GenBank: XP_ 014260029.1). Multiple alignments of sequences were performed using the multiple alignment program Clustal $\mathrm{W}$ in MEGA version 6.06. ${ }^{14}$ Phylogenetic analysis was performed by the neighbor-joining method using MEGA version 6.06 software. ${ }^{14}$ The reliability of the trees was tested by the bootstrap procedure with 1,000 replications.

\section{RESULTS AND DISCUSSION}

A number of behaviors within the triatomines life cycle are controlled by the circadian clock such as egg hatching, oviposition, ecdysis, sensitivity to light, thermopreference, and light/dark adaptation of compound eyes. ${ }^{6-11}$ In $R$. prolixus, four groups of photosensitive clock cells were found expressing the clock proteins PERIOD (PER) and TIMLESS (TIM) rhythmically. ${ }^{15}$ Their clock neurons are probably homologous to the side and dorsal neurons of $D$. melanogaster and are possibly responsible for controlling the rhythms in locomotor activity. ${ }^{16}$ Lateral neurons of $R$. prolixus exhibit robust rhythms in the abundance of the PER and TIM proteins with a peak in the dark phase. ${ }^{17}$ This clock receives outside information and generates rhythmicity in the release of neurohormones as prothoracicotropic hormone, providing temporal information to the peripheral oscillators. $^{18}$
The comparative analysis among the deduced amino acid sequences from $R$. prolixus and other insect species revealed conserved sites and domains typical of TIM protein (Timeless pfam04821 domain, PER interaction domains 1 and 2, and the amino acids corresponding to the nuclear and the cytoplasmic localizations). Phylogenetic analysis was performed based on the complete amino acid sequences of TIM protein of the hemipteran $R$. prolixus, C. lectularius, and $H$. halys, and of eight representative species of the orders Diptera, Lepidoptera, and Coleoptera. As expected, insect TIM sequences from the same insect order were grouped together with significant bootstrap support (Figure 1). Within Hemiptera, $R$. prolixus clustered with $C$. lectularius with high bootstrap support (100\%), as well as these two species with $H$. halys.

Similar to that observed for the clock gene per, the expression of the tim gene showed a daily canonical rhythm in the $T$. infestans group maintained under photoperiod (LD). In this group, the tim transcript level showed a significant increase at ZT12 and ZT16 in both sexes (Figure 2A and B). ${ }^{12}$ The JTK Cycle analyses indicated a significant rhythm in the expression of tim transcript in both sexes (adjusted $P$-value for females: 0.00050 and males: 0.0026 ), with the same period of 28 hours. However, differences in tim transcript rhythmic expression were detected in the amplitude (AMP) and phase (LAG). Females showed a lower amplitude and a higher phase (AMP: 14, LAG: 0.13103) than males (AMP: 18, LAG: 0.06632). In the constant light group, no changes were detected in tim transcript levels at the ZTs analyzed (Figure $2 \mathrm{C}$ and D). The corresponding mRNA levels remain flat in constant light with relatively similar levels of expression in both sexes at all

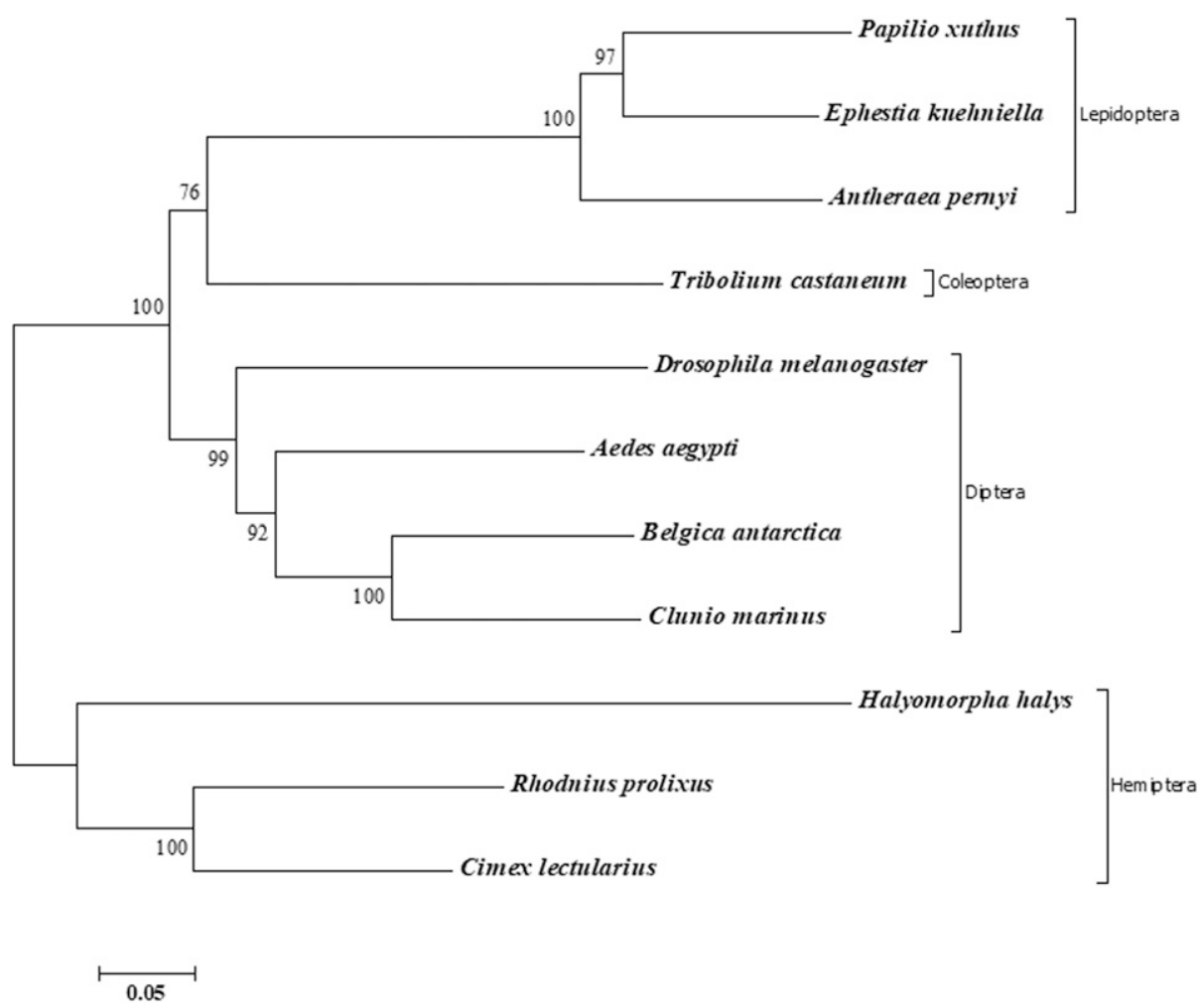

FIGURE 1. Neighbor-joining phylogeny of Rhodnius prolixus TIM deduced amino acid sequences, selected TIMs from other hemipteran species and representative species of the orders Lepidoptera, Coleoptera, and Diptera. Bootstrap values next to nodes represent the percentage of 1,000 replicate trees that preserved the corresponding clade. 
A

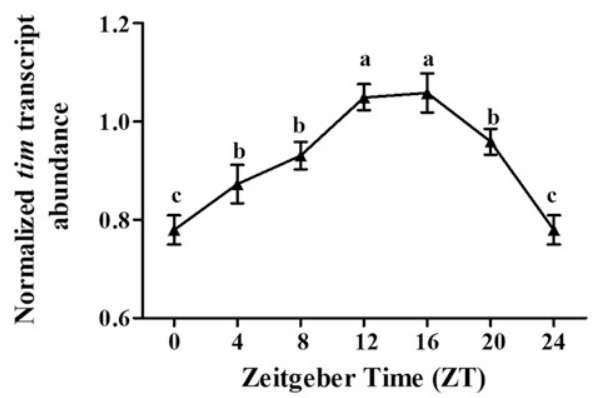

C

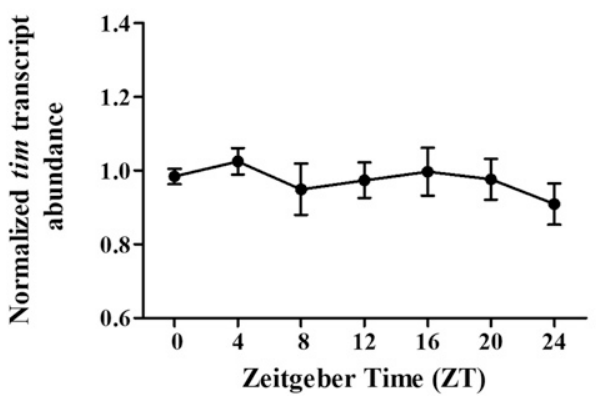

B

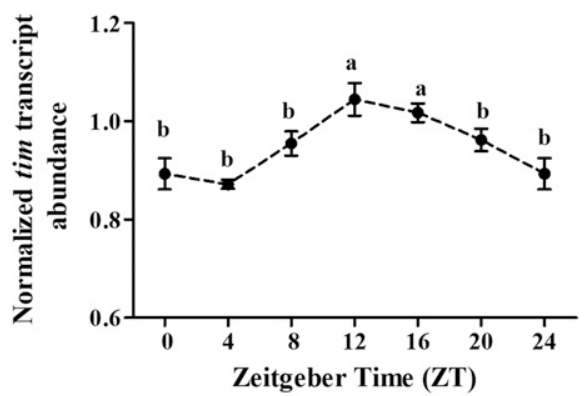

D

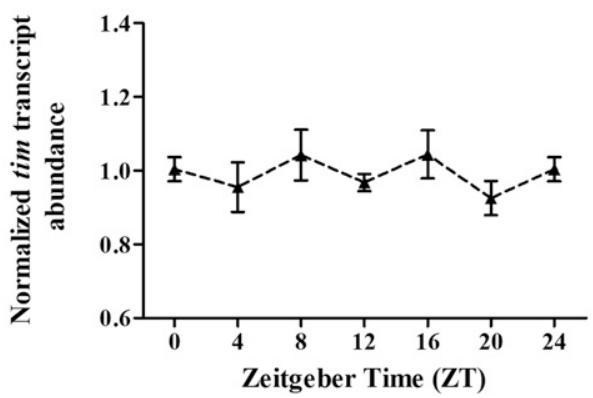

FIGURE 2. Circadian expression of tim gene in the nervous tissue of Triatoma infestans adults under photoperiod (A and $\mathbf{B})$ and constant light (C and D) conditions. Relative abundance of tim mRNA expression was referred to the ratio of tim mRNA vs. $\beta$-actin mRNA expression at each time point. Continuous and dash lines correspond to male and female groups, respectively. The error bars represent the SD of the mean. Different letters above the error bars indicate statistical differences determined by one-way analysis of variance followed by Bonferroni test $(P<0.05)$.

examined time points. The pattern of per and tim genes expression under LD, observed in adult females and males of $T$. infestans, agrees with the expression profile of this canonical clock gene in $D$. melanogaster. In this species, the mechanism of the circadian clock has been extensively studied and it has been observed that the clock genes are expressed in cycles close to 24 hours. ${ }^{5}$ Moreover, in $R$. prolixus, an increase in levels of PER and TIM proteins in the central clock neurons during the dark phase of the photoperiod was detected. ${ }^{15-17}$ Because $R$. prolixus and $T$. infestans are close species, it could be inferred that the increased level of per and tim mRNAs detected in T. infestans at sunset would promote a peak of PER and TIM protein levels at night, necessary for the function of the period/timeless loop in the clock cells. On the other hand, as expected, in the nervous tissue of the group maintained in constant light (LL), no daily increase was detected in per and tim transcript levels. The rhythms of expression of the tim and per genes showed visible damping in the LL group, confirming that prolonged exposure to light results in the loss of rhythmicity in the expression of these clock genes. The same effect of the light was demonstrated in studies about the levels of the PER and TIM proteins in clock neurons of $R$. prolixus. ${ }^{17}$

This kind of study would provide potentially useful information to analyze the temporal regulation of important biological processes, such as reproduction, dispersal, and insecticide resistance. For example, in adult mosquito $A e$. aegypti, the major vector of dengue viruses in Taiwan, daily fluctuation of insecticide resistance was found. Existence of a clock control over sensitivity to insecticide was further indicated by reduced expression of a cytochrome $P 450$ (CYP9M9), involved in detoxification metabolism, and reduced mosquito resistance to insecticide after temporal silencing of the per gene. These data provide the evidence on the circadian control of insect resistance to insecticides. ${ }^{19}$ Because it was also detected that daily variations in the expression of genes related to insecticide resistance in $T$. infestans, the clock genes could also be involved in the circadian control of the metabolic pathways of insecticide detoxification and resistance. ${ }^{20}$ Therefore, studies of circadian rhythms in Chagas disease vectors and their molecular basis, as well as the analysis of the central clock relationship with insecticide resistance, would promote the development of new control strategies.

Received February 27, 2019. Accepted for publication September 5, 2019.

Published online October 7, 2019.

Acknowledgments: We thank the Centro de Referencia de Vectores, Servicio Nacional de Chagas de Córdoba (Córdoba, Argentina), for providing insects used in our studies. This research was supported by grants from Agencia Nacional de Promoción Científica y Tecnológica (FONCyT-Argentina), Consejo Nacional de Investigaciones Científicas y Técnicas (CONICET-Argentina), and Secretaría de Ciencia y Tecnología, Universidad Nacional de Córdoba (SECyT-UNC-Argentina). M. M. S. and B. A. G. are career investigators of CONICET.

Authors' addresses: María M. Stroppa and Beatriz A. García, Instituto de Investigaciones en Ciencias de la Salud (INICSA), CONICET and Cátedra de Bioquímica y Biología Molecular, Facultad de Ciencias Médicas, Universidad Nacional de Córdoba, Córdoba, Argentina, E-mails: mercedesstroppa@hotmail.com and bgarcia@biomed.uncor.edu.

\section{REFERENCES}

1. Mougabure-Cueto G, Picollo MI, 2015. Insecticide resistance in vector Chagas disease: evolution, mechanisms and management. Acta Trop 149: 70-85. 
2. Boothroyd C, Wijnen H, Naef F, Saez L, Young LW, 2007. Integration of light and temperature in the regulation of circadian gene expression in Drosophila. PLoS Genet 3: e54.

3. Xu K, Zheng X, Sehgal A, 2008. Regulation of feeding and metabolism by neuronal and peripheral clocks in Drosophila. Cell Metab 8: 289-300.

4. Levine JD, Funes P, Dowse HB, Hall JC, 2002. Resetting the circadian clock by social experience in Drosophila melanogaster. Science 298: 2010-2012.

5. Hardin PE, 2011. Molecular genetic analysis of circadian timekeeping in Drosophila. Adv Genet 74: 142-158.

6. Ampleford Davey EJ, Davey KG, 1989. Egg laying in the insect Rhodnius prolixus is timed in a circadian fashion. $J$ Insect Physiol 35: 183-187.

7. Barrozo RB, Schilman PE, Minoli SA, Lazzari CR, 2004. Daily rhythms in disease-vector insects. Biol Rhythm Res 35: 79-92.

8. Lazzari CR, 1991. Circadian rhythm of egg hatching in Triatoma infestans (Hemiptera: Reduviidae). J Med Entomol 28: 740-741.

9. Lazzari CR, 1992. Circadian organization of locomotion activity in the haematophagous bug Triatoma infestans. J Insect Physiol 38: 895-903.

10. Minoli SA, Lazzari CR, 2003. Chronobiological basis of thermopreference in the haematophagous bug Triatoma infestans. J Insect Physiol 49: 927-932.

11. Lorenzo Figueiras AN, Kenigsten A, Lazzari CR, 1994. Aggregation in the haematophagous bug Triatoma infestans: chemical signals and temporal pattern. J Insect Physiol 40: 311-316.

12. Stroppa MM, Gimenez I, Garcia BA, 2018. Clock gene period in the Chagas disease vector Triatoma infestans (Hemiptera: Reduviidae). Am J Trop Med Hyg 98: 468-474.
13. Hughes ME, Hogenesch JB, Kornacker K, 2010. JTK CYCLE: an efficient nonparametric algorithm for detecting rhythmic components in genome-scale data sets. J Biol Rhythm 25: 372-380.

14. Tamura K, Stecher G, Peterson D, Filipski A, Kumar S, 2013. MEGA6: molecular evolutionary genetics analysis version 6.0. Mol Biol Evol 30: 2725-2729.

15. Steel CGH, Vafopoulou $X, 2006$. Circadian orchestration of developmental hormones in the insect, Rhodnius prolixus. Comp Biochem Physiol A Mol Integr Physiol 144: 351-364.

16. Helfrich-Förster C, 2004. The circadian clock in the brain: a structural and functional comparison between mammals and insects. J Comp Physiol A Neuroethol Sens Neural Behav Physiol 190: 601-613.

17. Vafopoulou $X$, Terry KL, Steel CG, 2010. The circadian timing system in the brain of the fifth larval instar of Rhodnius prolixus (Hemiptera). J Comp Neurol 518: 1264-1282.

18. Vafopoulou X, Steel CG, 2014. Synergistic induction of the clock protein PERIOD by insulin-like peptide and prothoracicotropic hormone in Rhodnius prolixus (Hemiptera): implications for convergence of hormone signaling pathways. Front Physiol 5: 1-12.

19. Yang YY, Liu Y, Teng HJ, Sauman I, Sehnal F, Lee HJ, 2010. Circadian control of permethrin-resistance in the mosquito Aedes aegypti. J Insect Physiol 56: 1219-1223.

20. Varela GM, Stroppa MM, García BA, 2019. Daily variations in the expression of genes related to insecticide resistance in the Chagas disease vector Triatoma infestans (Hemiptera: Reduviidae). Am J Trop Med Hyg 100: 1482-1485. 